\title{
Environmental allergens house dust mite-induced asthma is associated with ferroptosis in the lungs
}

\author{
WEIFENG TANG ${ }^{1,2^{*}}$, MING DONG ${ }^{3 *}$, FANGZHOU TENG $^{1,2}$, JIE CUI $^{1,2}$, \\ XUEYI ZHU ${ }^{1,2}$, WENQIAN WANG ${ }^{1,2}$, TULAKE WUNIQIEMU ${ }^{1,2}$, JINGJING QIN $^{1,2}$, \\ LA YI $^{1,2}$, SHIYUAN WANG ${ }^{1,2}$, JINGCHENG DONG $^{1,2}$ and YING WEI ${ }^{1,2}$
}

${ }^{1}$ Department of Integrative Medicine, Huashan Hospital, Fudan University; ${ }^{2}$ Cell and Molecular Biology Laboratory, Institutes of Integrative Medicine, Fudan University, Shanghai 200040; ${ }^{3}$ Department of Acupuncture and Orthopedics, Gumei Community Health Center, Shanghai 201102, P.R. China

Received May 18, 2021; Accepted September 9, 2021

DOI: $10.3892 /$ etm.2021.10918

\begin{abstract}
Previous studies have indicated that allergens such as house dust mites (HDM) in the environment can induce allergic asthma. Ferroptosis is a newly discovered form of regulatory cell death characterized by aberrant lipid peroxidation and the accumulation of reactive oxygen species (ROS) in cells. However, whether ferroptosis participates in the pathological process of asthma remains to be elucidated. The present study used a HDM-induced mouse asthma model to determine the effect of HDM exposure on allergic asthma and its underlying mechanisms. Female BALB/c mice were intranasally exposed to HDM to induce allergic asthma. Airway hyperresponsiveness (AHR), lung inflammation, mucus secretion, IgE levels, cytokine levels and inflammatory cell counts in bronchoalveolar lavage fluid (BALF) were investigated. In addition, the morphological changes of mitochondria, ROS levels, glutathione (GSH) levels and changes in ferroptosis pathway proteins were also determined in murine lungs. As a result, HDM exposure significantly increased AHR, inflammatory cell infiltration and mucus secretion around the airways. Furthermore, elevated IgE levels in the BALF, lung eosinophilia and a concomitant increase in IL-13 and IL-5 levels in BALF were observed. HDM inhalation increased ROS and decreased GSH levels in the lungs. HDM inhalation induced dysmorphic small mitochondria with decreased crista, as well as condensed, ruptured outer membranes. Western blotting demonstrated
\end{abstract}

Correspondence to: Professor Jingcheng Dong or Professor Ying Wei, Department of Integrative Medicine, Huashan Hospital, Fudan University, 12 Middle Urumqi Road, Shanghai 200040, P.R. China

E-mail: jcdong2004@126.com

E-mail: weiying_acup@126.com

${ }^{*}$ Contributed equally

Key words: house dust mites, allergic asthma, ferroptosis, reactive oxygen species, glutathione peroxidase 4 that the activities of glutathione peroxidase 4 and catalytic subunit solute carrier family 7 member 11 were significantly decreased, and that protein expression levels of acyl-CoA synthetase long-chain family member 4 and 15 lipoxygenase 1 were upregulated compared with mice in the normal control group. Overall, these results indicated that the AHR, airway inflammation, lipid peroxidation and ROS levels increased in HDM-induced asthma, and that HDM inhalation induced ferroptosis in the lungs, which helped to form an improved understanding of the pathogenesis of allergic asthma.

\section{Introduction}

Allergic diseases are experienced worldwide, with various risk factors and triggers that occur according to geographical and environmental differences (1). Allergic asthma is a heterogeneous inflammatory lung disease, characterized by lung inflammation, airway hyperresponsiveness (AHR) and airway remodeling (2). Statistically, $\sim 300$ million individuals worldwide suffer from asthma (3), half of which are allergic, with the incidence continuously increasing. Inhaled corticosteroids and long-acting bronchodilators are the main therapeutic drugs for clinical asthma treatment (4). However, the available treatments are often ineffective due to the heterogeneity of asthma and the variability of patient response to the available medications (5). Furthermore, certain patients have poor symptom control and suffer from recurrent exacerbations despite strictly adhering to therapy (6). Therefore, further mechanistic insights into the pathogenesis of asthma and targeted treatment strategies are required.

Environmental allergens, such as house dust mites (HDM), are major risk factors for asthma (7). HDM are commonly present in human dwellings and are especially abundant in mattresses, sofas, carpets and blankets (8). HDM can also be detected in the air, and studies using volumetric samples equipped with sizing devices have revealed that mite allergens remain airborne for a short period of time (9). Dermatophagoides pteronyssinus (of the Pyroglyphidae family), usually called Der $p$, is one of the main components in HDM; the first identified allergen was named Der $\mathrm{p} 1$, which is a glycoprotein (10). Sensitization to HDM is a major 
independent risk factor for asthma in areas where the climate is conducive to mite growth, especially in the tropical and subtropical regions (11). It has been suggested that mite fecal pellets may occasionally enter the lung and cause inflammation and bronchoconstriction $(11,12)$. Previous results have demonstrated that certain HDM allergens may have a direct effect on bronchial epithelia, inducing inflammation through IgE-independent mechanisms (12).

Ferroptosis, which was originally observed in 2003 and formally defined by Brent Stockwell, is an iron-dependent, non-apoptotic cell-death modality characterized by the accumulation of lipid hydroperoxides and lipid reactive oxygen species (ROS) in cellular membranes $(13,14)$. Ferroptosis is associated with mitochondrial fragmentation and cristae decrease (15). Ferroptotic cells do not exhibit any hallmarks of apoptosis or necroptosis, and are therefore identified through the balance between iron accumulation-induced ROS production and the antioxidant system that avoids lipid peroxidation (16). Glutathione peroxidase 4 (GPX4) is the major protective mechanism against peroxidation damage (17). Deprivation of glutathione (GSH) can inactivate GPX4, and therefore induce ferroptosis (18). Organs such as the kidneys, brain, liver, heart and lung are reported to be highly susceptible to ferroptosis under pathological conditions (15). Ferroptosis is also implicated in multiple human diseases, including Huntington's disease and diffuse large B cell lymphoma. However, whether ferroptosis participates in the pathological progression of asthma requires further elucidation. The present study used a HDM-induced mouse asthma model to determine the effect of HDM exposure on allergic asthma and the underlying mechanisms associated with ferroptosis.

\section{Materials and methods}

Animals and study protocol. In total, 20 female (6-8 week old, $20 \pm 2 \mathrm{~g}$ ), specified-pathogen free BALB/c mice were purchased from Shanghai SLAC Laboratory Animal Co., Ltd. Mice were kept under laboratory conditions $\left(22^{\circ} \mathrm{C} ; 50-60 \%\right.$ relative humidity; air circulation; 12-h light-dark cycle with free access to water and food). The experimental procedures were approved by the Ethics Committee of Animal Experiments of Fudan University (authorization no. 2020-10-HSYY-WY-01; Shanghai, China). Mice were randomized to two groups (10 mice/group): The normal control and HDM-induced asthma model groups. The asthma model was prepared as previously described $(19,20)$ and was modified according to pre-experimental results. On day 0 , HDM extract $(10 \mu \mathrm{g} / 40 \mu \mathrm{l})$ was administered intranasally after anesthetization with $2-2.5 \%$ isoflurane in air. From days $7-11$, the mice were challenged daily by pipetting $40 \mu \mathrm{l}$ diluted HDM extract (20 $\mu \mathrm{g} / 40 \mu \mathrm{l}$ per mouse) directly into the nostrils under mild anesthesia (Fig. 1A). For mice in the normal control group, the same volume of PBS was administered intranasally at the exact time as aforementioned.

In the present study, AHR levels, leukocyte differential counts in bronchoalveolar lavage fluid (BALF), inflammatory cytokines and histology were assessed to determine whether the asthma model was successfully established. Mice with outliers (One mouse in the control group was removed and two mice in the model group were removed) $(21,22)$, including abnormal values of airway resistance $\left(\mathrm{R}_{\mathrm{L}}\right)$, hematoxylin and eosin $(\mathrm{H} \& \mathrm{E})$ staining, periodic acid Schiff (PAS) staining and leukocyte differential counts in BALF, were considered to have failed the modeling process, and all the values were removed from the subsequent statistical analysis.

Reagents. Methacholine (Mch) and pentobarbital sodium were purchased from Sigma-Aldrich (Merck KGaA). HDM extract (Dermatophagoides pteronyssinus) was purchased from Greer Laboratories, Inc. The mouse lung dissociation kit was purchased from Miltenyi Biotec, Inc. Live eFluor780 (cat. no. 65-0865-14; 1:200 dilution) solution and anti-mouse CD45 eFluor 506 (cat. no. 69-0451-82; 1:100 dilution) antibodies were purchased from Thermo Fisher Scientific, Inc. Anti-mouse CD11b BV711 (cat. no. 563168, 1:100 dilution) and anti-mouse SiglecF BV421 (cat. no. 562681, 1:100 dilution) antibodies were purchased from BD Pharmingen (BD Biosciences). Anti-mouse Ly6C PE (cat. no. 128007, 1:200 dilution) antibodies were purchased from BioLegend, Inc. Anti-GPX4 (cat. no. Ab125066; 1:1,000 dilution) and anti-15 Lipoxygenase 1 (15-LO1; cat. no. Ab244205; 1:1,000 dilution) antibodies were purchased from Abcam, and anti-acyl-CoA synthetase long-chain family member 4 (ACSL4; cat. no. NBP2-16401; 1:1,000 dilution) and anti-catalytic subunit solute carrier family 7 member 11 (SLC7A11; cat. no. NB300-318; 1:1,000 dilution) antibodies were purchased from Bio-Techne. The GSH assay kit (cat. no. A006-2) was purchased from Nanjing Jiancheng Bioengineering Institute and ROS was measured using dihydroethydium (DHE; Sigma-Aldrich; Merck KGaA) oxidation. IgE [cat. no. 70-EK275-96; Multi Sciences (Lianke) Biotech, Co., Ltd.], IL-5 [cat. no. 70-EK205-96; Multi Sciences (Lianke) Biotech, Co., Ltd.] and IL-13 ELISA kits [cat. no. 70-EK213/2-96; Multi Sciences (Lianke) Biotech, Co., Ltd.] were purchased from Hangzhou Multi Sciences (Lianke) Biotech Co., Ltd. RIPA lysis buffer was purchased from Beyotime Institute of Biotechnology. The BCA protein test kit was purchased from Thermo Fisher Scientific, Inc. and the Immobilon Western Chemiluminescent HRP Substrate was purchased from Merck KGaA.

Measurement of AHR. Within $24 \mathrm{~h}$ after the final HDM challenge, AHR presented as $\mathrm{R}_{\mathrm{L}}$ and dynamic lung compliance (Cdyn) to Mch were measured according to the manufacturer's protocols (DSI Buxco ${ }^{\circledR}$ FinePointe Resistance and Compliance system; Data Sciences International; Harvard Bioscience, Inc.). Mice were weighed, intraperitoneally anesthetized with pentobarbital sodium $(50 \mathrm{mg} / \mathrm{kg})$, incised and intubated via the trachea. Afterwards, mice were placed into the chamber of the Resistance and Compliance system at room temperature and the tracheal intubation was connected to a ventilator. AHR was challenged with increasing doses of Mch $(0,6.25,12.5$, 50 or $100 \mathrm{mg} / \mathrm{ml}$; Each dose has a 3-min response time and there was a 1-min interval between each dose) and data was recorded and presented as changes in $\mathrm{R}_{\mathrm{L}}$ and Cdyn.

Histological analysis. Anesthetized mice were sacrificed by cervical dislocation following AHR detection. Intact left and both upper and lower lobes of the right lung were snap frozen in liquid nitrogen $\left(-196^{\circ} \mathrm{C}\right)$ and then stored at $-80^{\circ} \mathrm{C}$ until further use. The middle lobe of the right lung was fixed in $4 \%$ paraformaldehyde at room temperature for $24 \mathrm{~h}$ and used for subsequent histological analysis. H\&E and PAS 
staining were performed to determine the inflammatory and mucus secretion changes. The middle lobe of right lung from each mouse was removed, fixed in $4 \%$ paraformaldehyde at room temperature for $24 \mathrm{~h}$, decalcified in EDTA, dehydrated in a graded series of ethanol solutions and embedded in paraffin. Afterwards, lung tissue sections were obtained at a thickness of 4- $\mu \mathrm{m}$. Lung slices were stained with H\&E (hematoxylin staining for $10 \mathrm{~min}$ at room temperature; eosin staining for $3 \mathrm{~min}$ at room temperature) and PAS solution and imaged with light microscopy (x100 or x200, magnifications; Nikon ECLiPSE Ni; Nikon Corporation). A semi-quantitative grading method was used to evaluate the degree of peribronchial inflammation as described by Myou et al (23). The severity of the inflammation was graded in five categories: 0 , Normal; 1 , few inflammatory cells; 2 , a ring of inflammatory cells one cell layer deep; 3, a ring of inflammatory cells two to four cells deep; and 4, a ring of inflammatory cells of four cells deep. PAS-positive cells in each airway were counted, divided by the circumference of the basement membrane and multiplied by 100 (24). The data was collected by three independent blinded investigators (FZT, JC, JJQ).

Transmission electron microscopy (TEM). Changes in mitochondrial structure were observed using TEM. Lower lobe of right lung was cut into $1 \times 1 \times 3 \mathrm{~mm}^{3}$ sections and fixed in $2.5 \%$ glutaraldehyde phosphate buffer at $4^{\circ} \mathrm{C}$ for $24 \mathrm{~h}$. Fixed samples were dehydrated with ethanol and acetone, embedded (Epon812; cat. no. 45345; Sigma-Aldrich; Merck KGaA) and dried. Afterward, the tissue was cut into 70-nm thick sections, stained with uranyl acetate and citrate at room temperature for 30 min and visualized using the JEM-1400 Plus transmission electron microscope (magnification, x20,000; JEOL, Ltd.).

Leukocyte differential counts in BALF. BALF was collected by flushing the right lungs three times with $1 \mathrm{ml}$ PBS using a 1-ml syringe inserted into a cannula. BALF was centrifuged for $10 \mathrm{~min}$ at $500 \mathrm{x} \mathrm{g}$ and $4^{\circ} \mathrm{C}$, after which the supernatant was collected for cytokines analysis. The pellet containing the cells was resuspend in $100 \mu \mathrm{l}$ PBS to determine the leukocyte differential count using the BC-5000 Vet auto hematology analyzer (MINDRAY Medical International Co., Ltd.).

Cytokines in BALF. IgE and T helper (Th) 2 cells play a pathogenic role in asthma (25). The levels of IgE, along with Th2 cytokines IL-5 and IL-13, were therefore investigated in the supernatants of BALF samples using sandwich ELISA kits according to the manufacturer's instructions.

Flow cytometry of lung tissue. Eosinophil (Eos) has been implicated in the pathogenesis of asthma (26). Eos populations in lung tissue were detected using flow cytometry. $\mathrm{CD} 5^{+} \mathrm{CD} 11 \mathrm{~b}^{+}$Ly6C-SiglecF ${ }^{+}$cells were identified as the EOS population as described by a previous study (27). The mouse lung dissociation kit was used to dissociate the upper right lobe of each lung to single cells. Afterwards, single cells were stained with the fluorescently labeled dyes or antibodies (Live eFluor780, CD45 eFluor 506, anti-mouse CD11b BV711, anti-mouse SiglecF BV421, anti-mouse Ly6C PE) at $1 \times 10^{6}$ cells $/ 100 \mu \mathrm{l}$ at $4^{\circ} \mathrm{C}$ for 30 min detected using an Attune
NxT instrument (Thermo Fisher Scientific, Inc.). Flow analysis was done using the FlowJo version 10 software (FlowJo, LLC).

ROS and GSH measurement. For ROS, the tissue samples were embedded with Tissue-Tek ${ }^{\circledR}$ OCT (cat. no. 4583; Sakura Finetek USA, Inc.) and then prepared into frozen sections. The embedded samples were cut into $10-\mu \mathrm{m}$ thick slices using a cryostat (CM1860; Leica Microsystems GmbH) and transferred onto glass slide and stored at $-20^{\circ} \mathrm{C}$. They were then stained according to the protocols of the ROS kit. For GSH sample preparation, the tissue sample was prepared into a $10 \%$ tissue homogenate [tissue weights (g): Volume of normal saline $(\mathrm{ml})=1: 9]$, and the subsequent determination was performed according to the protocols of the GSH kit. Ferroptosis is determined by the balance between iron accumulation-induced ROS production and the antioxidant system that avoids lipid peroxidation (16). In the present study, the upper lobe of the left lung is used for ROS and part of the lower right lung is used for GSH detection, ROS and GSH levels were determined using commercial ROS and GSH assay kits according to the manufacturer's instructions.

Western blot assay. GPX4, ACSL4, 15-LO1 and SLC7A11 are mediators of lipid peroxidation and ferroptosis, and have been reported to be involved in the ferroptosis pathway (16). The expression levels of these proteins were assayed using western blotting. Lung tissues were homogenized and sonicated in RIPA lysis buffer, and then centrifuged at 13,201 x $\mathrm{g}$ for $10 \mathrm{~min}$ at $4^{\circ} \mathrm{C}$. Afterwards, the supernatants were collected and the total protein level was measured using a BCA protein test kit. A $12 \%$ SDS-PAGE separation gel (30 $\mu \mathrm{g}$ per lane) was prepared for protein separation and transferred to PVDF membranes, followed by blockage in 5\% non-fat milk at room temperature for $1 \mathrm{~h}$. Afterwards, the membranes were washed three times with Tris buffered saline $0.1 \%$ Tween-20 every $10 \mathrm{~min}$. After that, the PVDF membranes were incubated with GPX4 $(1: 1,000)$, ACSL4 (1:1,000), 15-LO1 $(1: 1,000)$ and SLC7A11 $(1: 1,000)$ antibodies at $4^{\circ} \mathrm{C}$ overnight. After three washes with TBST, the PVDF membranes were incubated with secondary antibodies for $1.5 \mathrm{~h}$ at room temperature (goat anti-rabbit IgG HRP-conjugated secondary antibody; cat. no. L3012; Signalway Antibody LLC; 1:10,000 dilution). Immobilon western chemiluminescent HRP substrate solution was used to exhibit protein band after three washes with TBST. $\beta$-actin (1:10,000; dilution; cat. no. HRP-60008; Proteintech Group, Inc.) was used as the internal control for the normalization of the data using ImageJ (v152p; National Institutes of Health).

Statistical analysis. All the experiments were repeated $\geq$ three times. All data were analyzed and graphed using GraphPad Prism 8 (GraphPad Software, Inc.). Data are presented as the mean \pm standard deviation. Unpaired Student's t-test or Mann-Whitney U test were used to analyze the difference between two samples. $\mathrm{P}<0.05$ was considered to indicate a statistically significant difference.

\section{Results}

HDM exposure induces an increase in AHR following Mch treatment. Changes in AHR were investigated in the current 
A

Sensitizations

Challenges

Sacrifice

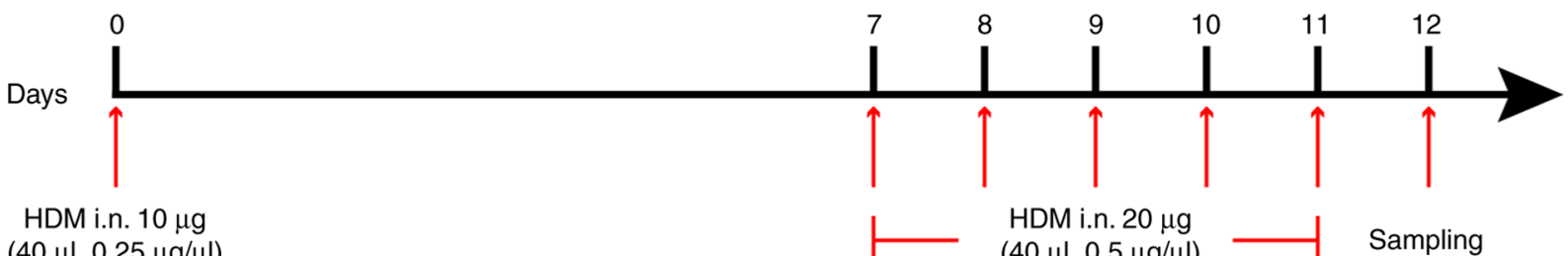

$(40 \mu \mathrm{l}, 0.25 \mu \mathrm{g} / \mu \mathrm{l})$

$(40 \mu \mathrm{l}, 0.5 \mu \mathrm{g} / \mu \mathrm{l})$

Sampling

$\mathrm{B}$

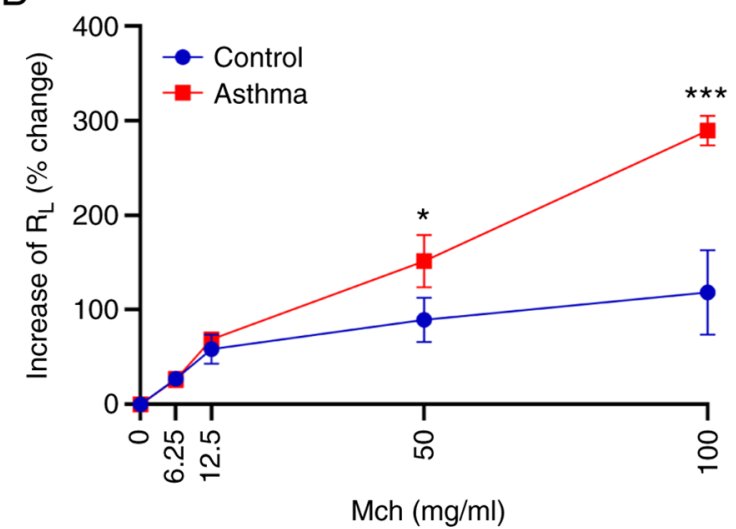

C

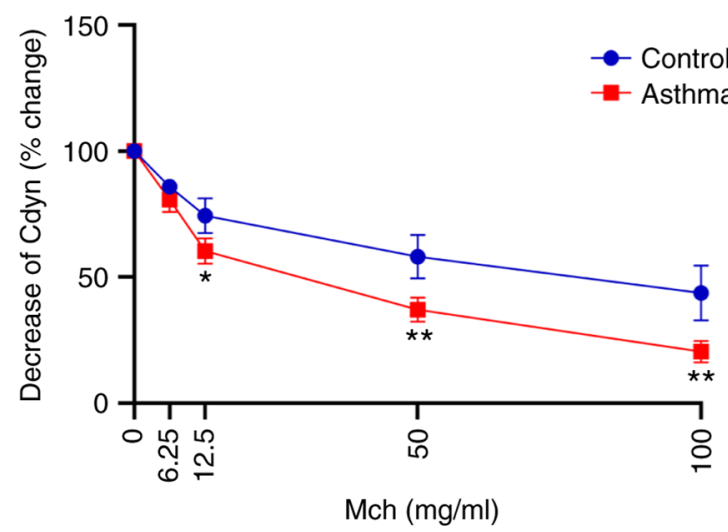

Figure 1. HDM exposure induces an increase in AHR. (A) Protocol of preparation of HDM-induced asthma model. Changes in (B) $\mathrm{R}_{\mathrm{L}}$ and (C) Cdyn are presented. ${ }^{*} \mathrm{P}<0.05,{ }^{* *} \mathrm{P}<0.01$ and ${ }^{* * * *} \mathrm{P}<0.001$ compared with the control. HDM, house dust mites; AHR, Airway hyperresponsiveness; $\mathrm{R}_{\mathrm{L}}$, airway resistance; Cdyn, dynamic lung compliance; Mch, methacholine.

study. Compared with mice in the normal control group, when the Mch dose increased, the $\mathrm{R}_{\mathrm{L}}$ increased significantly $(\mathrm{P}<0.05$ or $\mathrm{P}<0.001$; Fig. 1B), whereas Cdyn decreased significantly $(\mathrm{P}<0.05$ or $\mathrm{P}<0.01$; Fig. $1 \mathrm{C})$. There was no obvious increase in $\mathrm{R}_{\mathrm{L}}$ between the two groups at Mch doses $<50 \mathrm{mg} / \mathrm{ml}$ (Fig. 1B). However, Mch challenge at 50 and $100 \mathrm{ng} / \mathrm{ml}$ resulted in the prominent augmentation of $\mathrm{R}_{\mathrm{L}}$. At Mch doses of $12.5(\mathrm{P}<0.05)$, $50(\mathrm{P}<0.01)$ and $100(\mathrm{P}<0.01) \mathrm{mg} / \mathrm{ml}, \mathrm{HDM}$ inhalation induced a significant decrease in Cdyn compared with the control group (Fig. 1C). These data indicated that HDM exposure could aggravate AHR.

HDM exposure promotes lung inflammation and goblet cell hyperplasia. H\&E staining was performed to evaluate the inflammatory changes within the lung. The results revealed that inflammatory cell infiltration around the bronchus (Fig. 2A and B) was observed in mice receiving HDM inhalation, and that HDM exposure significantly aggravated the pulmonary inflammatory response compared with the control ( $\mathrm{P}<0.05$; Fig. 2B). Mucus hypersecretion is a notable feature of allergic asthma (28). Therefore, PAS staining was conducted to evaluate the degree of goblet cell metaplasia and mucus secretion. As presented in Fig. 2A and C, mucus secretion increased significantly compared with mice in the normal control group. In addition, inflammatory cells in BALF were also detected, and the results demonstrated that the total leukocytes, lymphocytes (Lym), Eos and monocytes (Mon) increased significantly after HDM exposure compared with the control $(\mathrm{P}<0.05$; Fig. 2D). These aforementioned data suggest that HDM exposure promoted lung inflammation and goblet cell proliferation.
HDM inhalation enhances airway eosinophilic and Thelper (Th)2-associated inflammation. $\mathrm{CD}^{4} 5^{+} \mathrm{CD} 11 \mathrm{~b}^{+}$SiglecF ${ }^{+} \mathrm{Ly} 6 \mathrm{C}$ in Eos populations of the lung tissue were detected using flow cytometry. Mice in the HDM-induced asthma group demonstrated significantly increased profiles of $\mathrm{CD} 45^{+} \mathrm{CD}_{11 \mathrm{~b}}{ }^{+}$SiglecF ${ }^{+} \mathrm{Ly} 6 \mathrm{C}$ in the Eos proportion compared with mice in the control group $(\mathrm{P}<0.01$; Fig. $3 \mathrm{~A}$ and $\mathrm{B})$. Furthermore, IgE, IL-5 and IL-13 levels in BALF were also investigated using ELISA. Elevated IgE, IL-5 and IL-13 expression levels were detected compared with control group mice $(\mathrm{P}<0.05, \mathrm{P}<0.01$ or $\mathrm{P}<0.001$; Fig. 3C-E). Therefore, HDM-induced airway inflammation was characterized by Th2-mediated eosinophilic inflammation in the lungs.

HDM exposure induces ferroptosis in the lung. Imbalance between ROS production and the antioxidant system is associated with ferroptosis (16). The present study revealed that HDM exposure resulted in significantly elevated ROS $(\mathrm{P}<0.05$; Fig. 4A and $\mathrm{B})$ and reduced GSH levels $(\mathrm{P}<0.05$; Fig. 4C) in the lung tissue compared with those in the control mice. It has been reported that ferroptosis is associated with mitochondrial fragmentation and cristae decrease (29). To explore the role of HDM inhalation in ferroptosis, the morphological changes of mitochondria were observed using TEM. Consistent with literature reports $(15,27,30)$, dysmorphic small mitochondria were observed in the lung cells after HDM inhalation. Representative results are presented in Fig. 4D. Decreased mitochondria crista, condensed and ruptured outer membranes were also discovered in the lung cells of mice in HDM-induced asthma group. These results provided evidence that HDM exposure could induce ferroptosis in the lungs. 
A

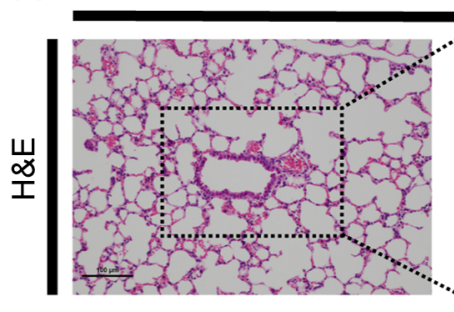

Control
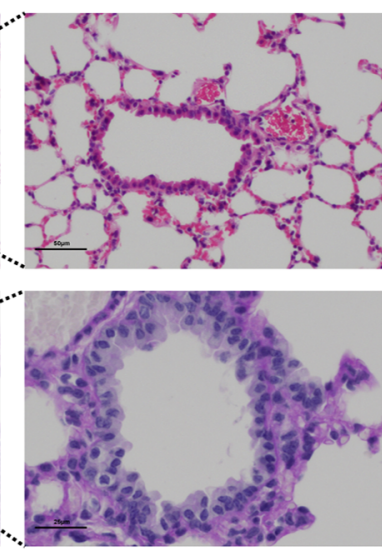

Asthma
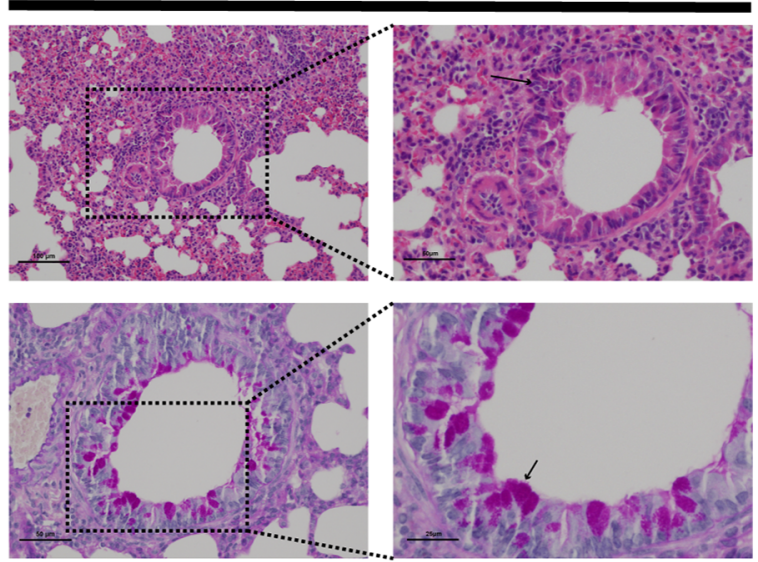

B

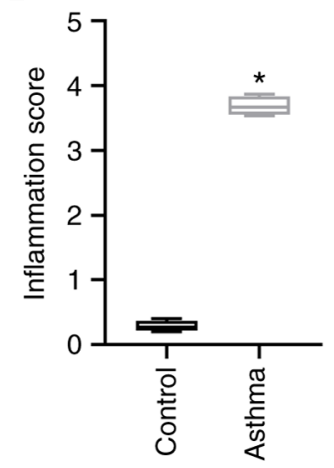

C

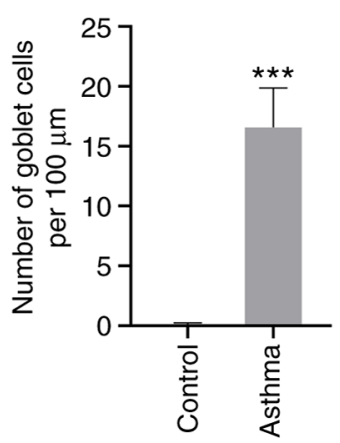

D

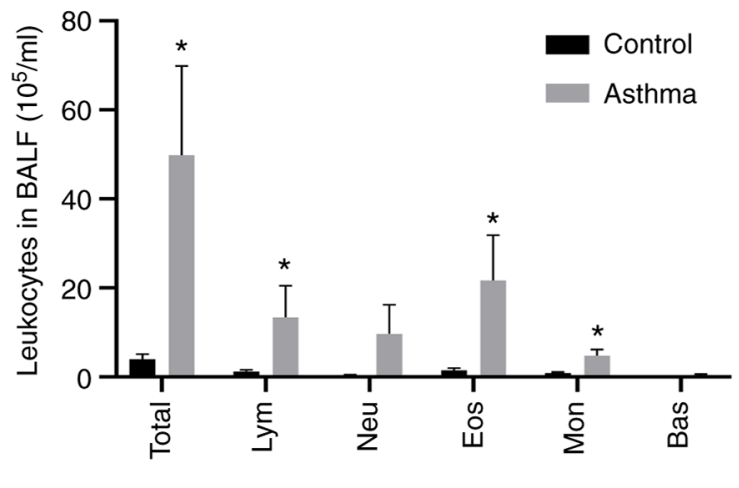

Figure 2. House dust mite exposure promotes lung inflammation and goblet cell hyperplasia. (A) Representative images of H\&E (x100, scale bars, $100 \mu$ m; x200, scale bars, $50 \mu \mathrm{m}$ ) and PAS staining (x200, scale bars, $50 \mu \mathrm{m}$; x400, scale bars, $25 \mu \mathrm{m}$ ) results (arrows indicate PAS-positive cells). (B) Inflammation score of the H\&E results. (C) Changes in goblet cell counts. (D) Classification of leukocytes in the BALF. ${ }^{*} \mathrm{P}<0.05$ and ${ }^{* * * *} \mathrm{P}<0.001$ compared with the control. H\&E, hematoxylin and eosin; PAS, periodic acid Schiff; BALF, bronchoalveolar lavage fluid; Lym, lymphocytes; Neu, Neutrophils; Eos, eosinophils; Mon, monocytes; Bas, basophils.

HDM exposure induces dysregulation of proteins associated with the ferroptosis pathway. To investigate the underlying mechanisms associated with ferroptosis, key proteins involved in ferroptosis were measured. The protein expression levels of GPX4, ACSL4, 15-LO1 and SLC7A11 were detected. The quantified western blotting results revealed that mice that received HDM inhalation had significantly downregulated activities of GPX4 compared with the normal control group $(\mathrm{P}<0.01$; Fig. 4E and G). Furthermore, a significant upregulation of ACSL4 and 15-LO1 as well as downregulation of SLC7A11 were detected in HDM-induced asthmatic mice compared with the controls ( $\mathrm{P}<0.05$; Fig. 4E, F, H and I). These results demonstrated that GPX4, ACSL4, 15-LO1 and SLC7A11 were involved in ferroptosis in the lungs of HDM-induced asthmatic mice.

\section{Discussion}

Asthma is a heterogeneous chronic inflammatory disease of the airways characterized by chronic airway inflammation, bronchoconstriction, AHR and mucus hypersecretion $(31,32)$. Previous studies have revealed that asthma is a heterogeneous disease with multiple phenotypes and endotypes $(33,34)$. Allergic asthma is the most common form of asthma, which can be triggered by allergens in the environment, such as HDM $(7,8)$. As a naturally occurring allergen in the environment, HDM is frequently used for the preparation of allergic mouse asthma models to uncover the key inflammatory pathways in the progress of allergic asthma (35).

The present study used an HDM-induced mouse asthma model to determine the effect of HDM exposure on allergic asthma and the mechanisms that underlie this. The results demonstrated that HDM administered intranasally increased AHR in mice compared with mice exposed to PBS. Asthma is characterized by chronic inflammation in the airways. In order to observe the inflammatory changes in the airways, $\mathrm{H} \& \mathrm{E}$ and PAS staining of the lung sections were performed in the current study. Inflammatory cell infiltration around the bronchus, goblet cell metaplasia and mucus hypersecretion were observed in murine lung tissue after HDM inhalation. Furthermore, the levels of total leukocytes, Lym, Eos and Mon increased significantly after HDM exposure, indicating that lung inflammation was induced by HDM. In addition,

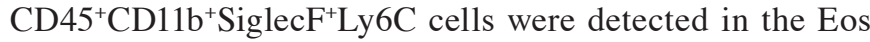
population of the lung tissue, which was consistent with the results of BALF leukocyte classification. Therefore, mice in the HDM-induced asthma group demonstrated significantly increased levels of $\mathrm{CD} 45^{+} \mathrm{CD}_{11 b^{+} \text {SiglecF }} \mathrm{Ly}_{6 \mathrm{C}}$ in the Eos population.

IgE and Th2 cells play a pathogenic role in asthma $(36,37)$. Thus, the present study further investigated the levels of $\operatorname{IgE}$ and 

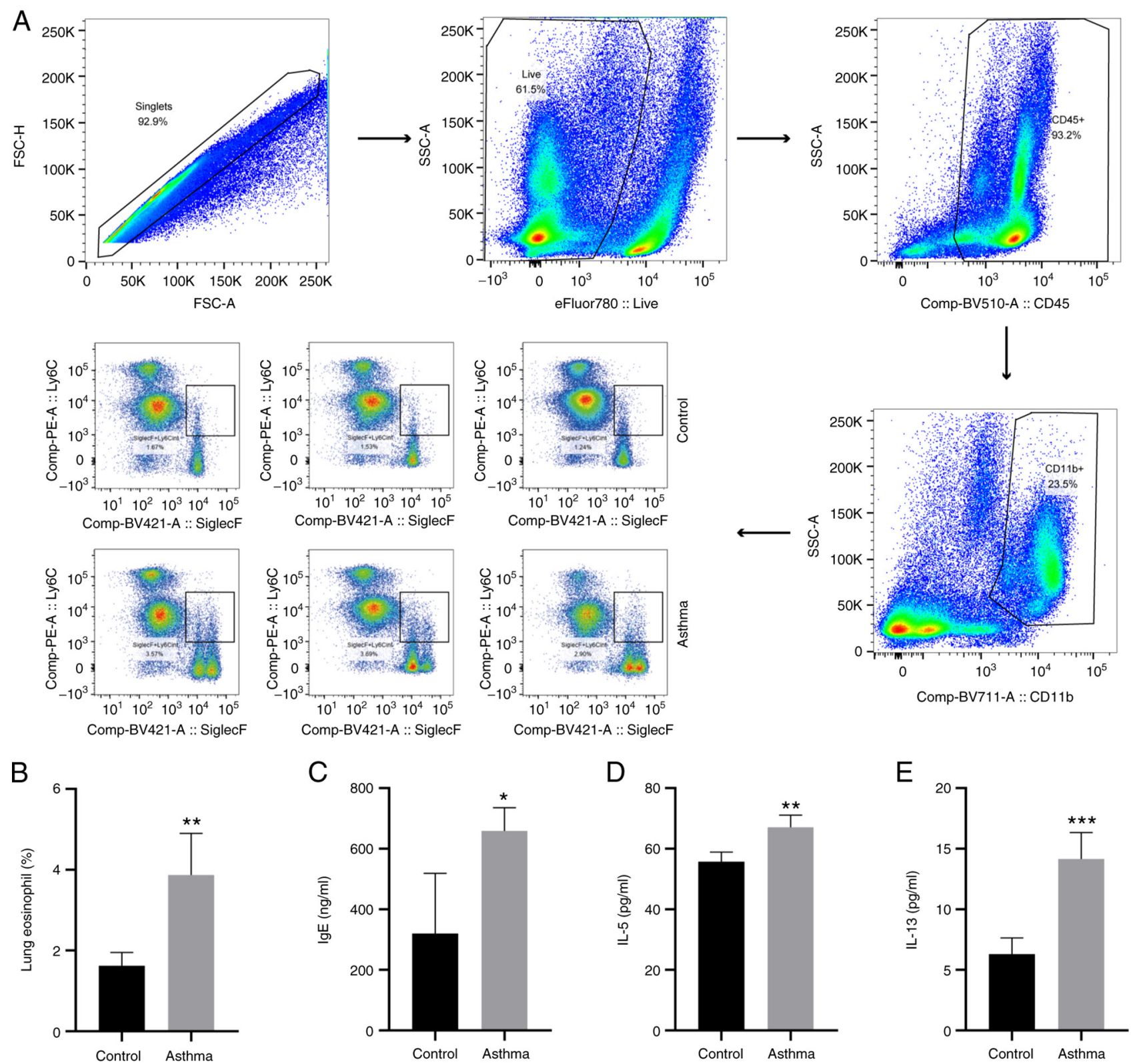

Figure 3. House dust mite exposure induces airway eosinophilic and T helper 2-associated inflammation. (A) Flow cytometry analysis of eosinophils in the lung. (B) Changes of $\mathrm{CD}_{4} 5^{+} \mathrm{CD}_{11 \mathrm{~b}}{ }^{+}$SiglecF $\mathrm{F}^{+} \mathrm{Ly} 6 \mathrm{C}$ in eosinophil proportions are presented using a bar graph. Relative expression levels of (C) IgE, (D) IL-5 and (E) IL-13 in BALF were assessed. ${ }^{*} \mathrm{P}<0.05,{ }^{* *} \mathrm{P}<0.01$ and ${ }^{* * * *} \mathrm{P}<0.001$ compared with the control group. BALF, bronchoalveolar lavage fluid.

Th2 cytokines IL-5 and IL-13 in the supernatant of BALF. It has been hypothesized that the cysteine protease activity of Der $\mathrm{p} 1$ in HDM selectively enhances the IgE response and upregulates $\mathrm{IgE}$ synthesis by cleaving the low-affinity IgE receptor (CD23) from the surface of human B cell lymphocytes (38). Allergic asthma accounts for $\sim 80 \%$ of asthma cases, and high levels of Th2 cytokines can be detected in lung tissue and BALF (39). Mounting evidence has demonstrated that IL-5 and IL-13 play a prominent role in eosinophil activation and mucus hyperplasia $(40,41)$. Consistent with these former reports, the present study revealed that mice exposed to HDM had elevated $\operatorname{IgE}$, IL-5 and IL-13 expression levels compared with mice receiving PBS. Overall, the results indicated that HDM inhalation induced AHR and type 2 airway inflammation in asthma.

Ferroptosis is an iron-dependent cell-death modality driven by oxidative phospholipid damage and has been implicated in a number of diseases, including Huntington's disease and diffuse large B cell lymphoma $(42,43)$. The current study investigated whether ferroptosis participated in the pathological progression of HDM-induced asthma. Mitochondria have been reported to be involved in cysteine-deprivation-induced ferroptosis (15). Morphological changes of mitochondria, which include mitochondrial fragmentation and a decrease in cristae, are observed in ferroptosis (29). Furthermore, certain potent ferroptosis inhibitors have been demonstrated to target mitochondria (44). A previous study demonstrated that lipid ROS is required for ferroptosis (14), indicating that the accumulation of ROS is a key feature of ferroptosis. Moreover, mitochondria are the major organelle of cellular ROS production. A decreased level of lipid ROS accumulation has been observed in mitochondria-depleted cells (15), which further confirms the involvement of mitochondria in 
A

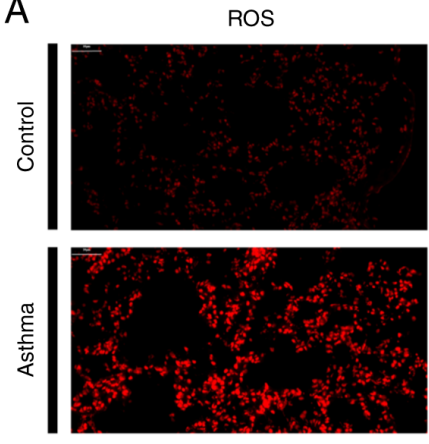

DAPI
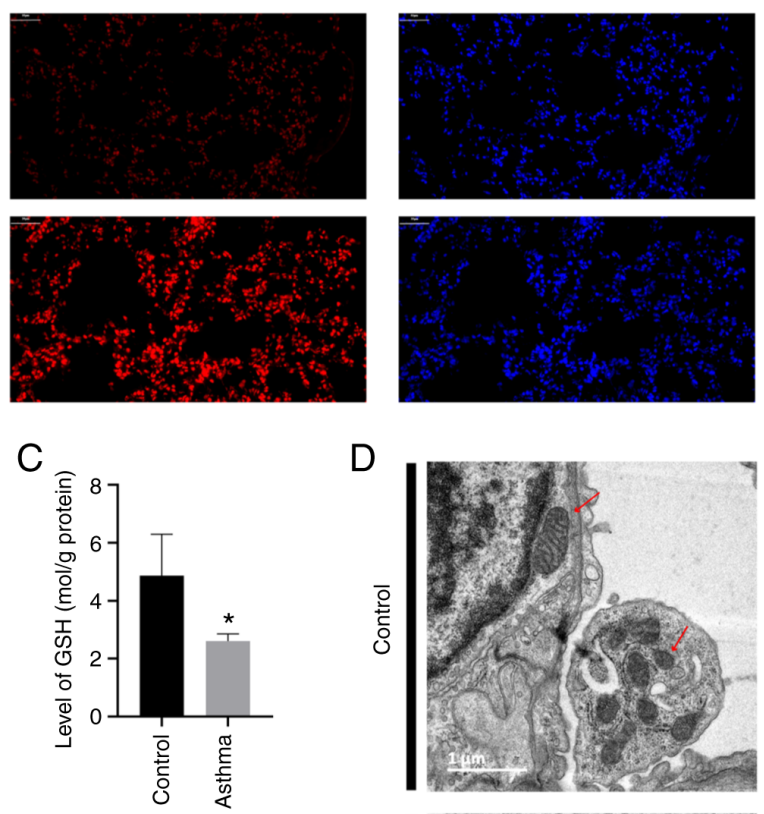

D

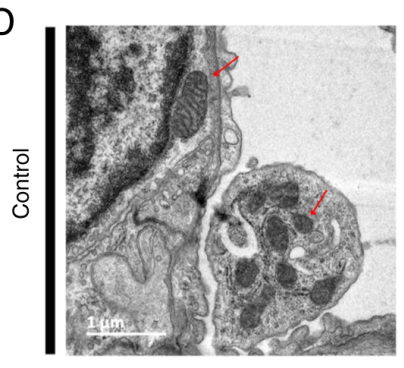

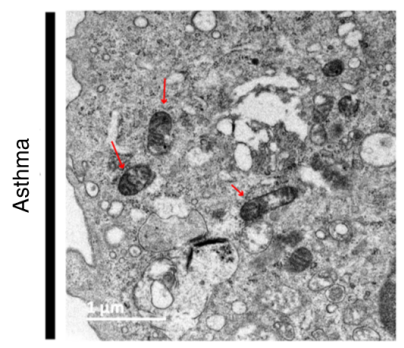

$\mathrm{F}$
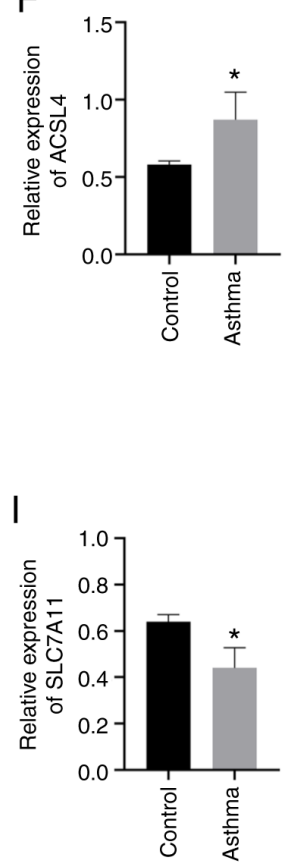

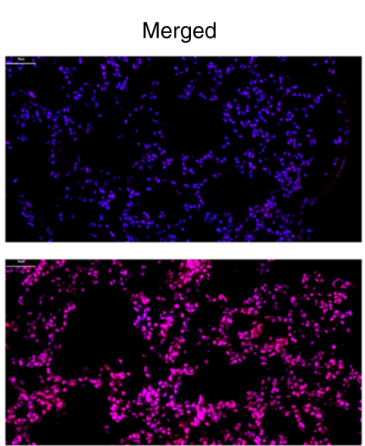

B
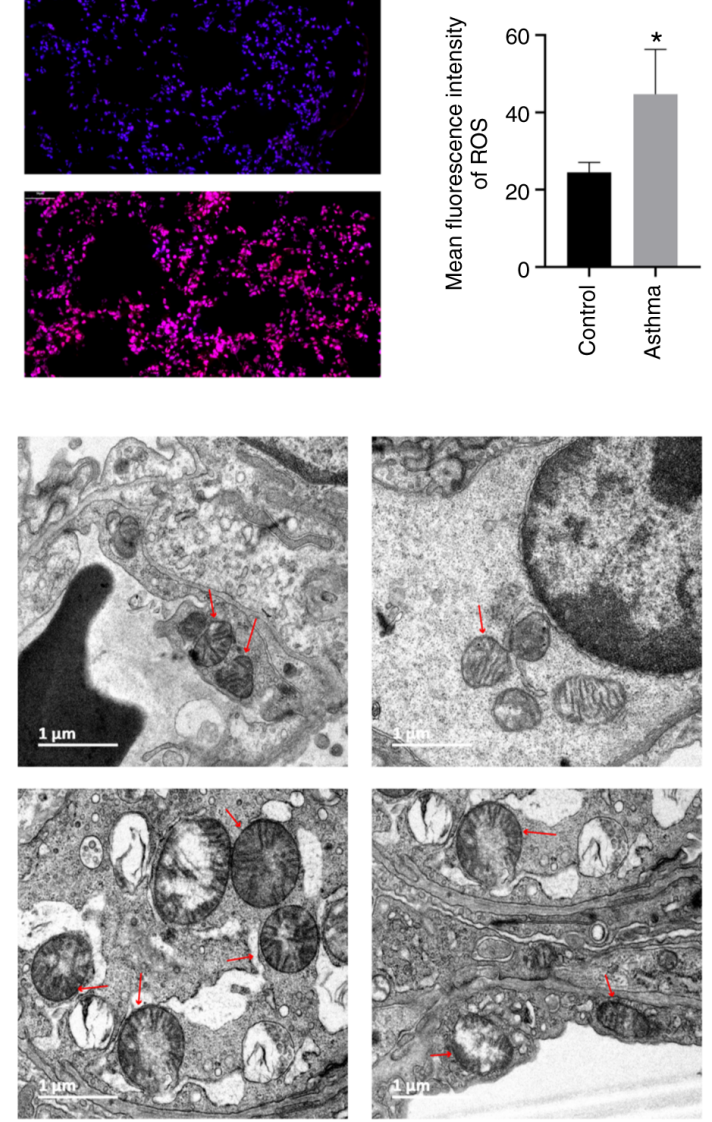

G

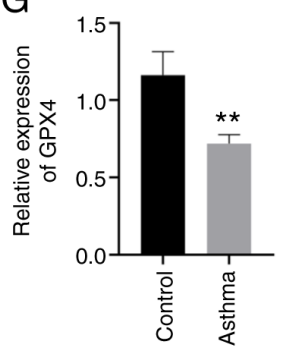

J $\quad y=\overbrace{\text { HDM Inhalation }}^{\text {Glutamate }}$ HDM Inhalation

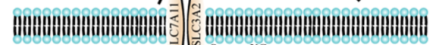

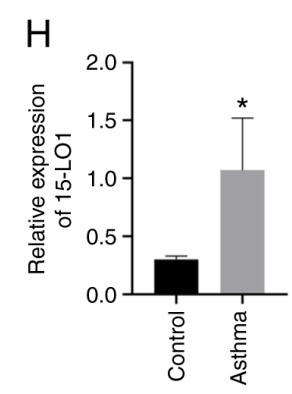

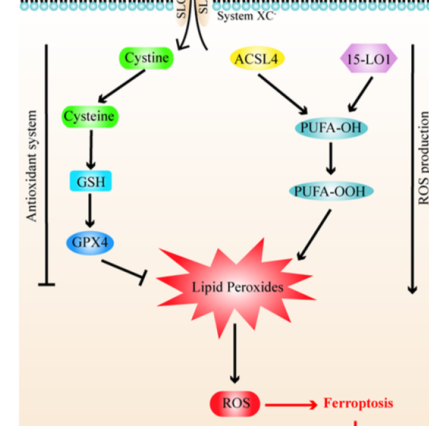

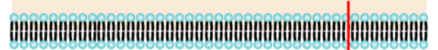

Figure 4. HDM exposure induces ferroptosis in the lungs. (A and B) ROS production in the lungs. (C) GSH level in the lung. (D) Morphological changes of mitochondria in ferroptosis after HDM exposure (arrows indicate mitochondria). (E) Representative image of western blotting of ferroptosis. Expression levels of (F) ACSL4, (G) GPX4, (H) 15-LO1 and (I) SLC7A11 in the lungs. (J) Pathways controlling ferroptosis in HDM-induced asthma pathogenesis. System Xc-, a cystine-glutamate antiporter. "P $<0.05,{ }^{* *} \mathrm{P}<0.01$. HDM, house dust mites; ROS, reactive oxygen species; GSH, glutathione; ACSL4, anti-acyl-CoA synthetase long-chain family member 4; GPX4, Glutathione peroxidase 4; 15-LO1, 15 Lipoxygenase 1; SLC7A11, solute carrier family 7 member 11; PUFA-OOH, polyunsaturated fatty acids; - $\mathrm{OOH}$, hydroperoxy radicals. 
ferroptosis. In the present study, morphological changes of mitochondria were observed using TEM, the results of which revealed that decreased mitochondria crista and condensed and ruptured outer membranes were observed in the lung cells of mice in the HDM-induced asthma group, which was consistent with previous results.

Polyunsaturated fatty acids (PUFAs) are the primary targets of lipid peroxidation on cell membranes (16). The activity of multiple upstream cascades determine the sensitivity to ferroptosis (including free PUFAs) as well as the balance between levels of pro-oxidant factors (ROS and lipoxygenases) and antioxidant factors (GPX4 and GSH) (45). GSH is an anti-oxidant that serves as a cofactor for the reduction of lipid hydroperoxides by GPX4 (46). It has been reported that depletion of GSH by erastin inactivates GPX4, leading to the accumulation of lipid ROS and lipid peroxidation (47). Inhibition of GSH synthesis induces ferroptosis in certain cells. In the present study, ROS and GSH levels in the lung were detected, and the results demonstrated that HMD exposure induced an increase of ROS production and reduced GSH levels in the lung tissue. This indicated that HDM exposure may result in an imbalance between pro-oxidant and anti-oxidant factors in the lung (26).

GPX4 is an enzyme required for the clearance of lipid ROS. GPX4 catalyzes GSH to eliminate the production of phospholipid hydroperoxides, which are major mediators of chain reactions in lipoxygenases (16). As the furthest downstream component of the ferroptosis pathway, inactivation of GPX4 results in ferroptosis even when cellular cysteine and GSH contents are normal (48). GPX4 activity was assessed by western blotting in the present study. The results revealed that mice receiving HDM inhalation had downregulated levels of GPX4 activity. ACSL4 functions to preferentially activate long PUFAs for phospholipid biosynthesis (29). The synthesis of long chain PUFA-CoA was inhibited in $\mathrm{ACSL}^{-/}$cells, supporting the hypothesis that of ACSL4 has a function in lipid biosynthesis (29). The upregulation of ACSL4, but not other ACSL members, is an important component for ferroptosis $(29,49)$. Resistance to ferroptosis was observed in GPX4/ACSL4 double knockout cells. Previous results indicated that ACSL4 inhibition can be used as a therapeutic approach to prevent ferroptosis-related diseases (50).

The present study also investigated the effect of HDM inhalation on ACSL4 expression. Upregulation of ACSL4 was observed in HDM-induced asthma, indicating the augmentation of lipid biosynthesis by HDM inhalation. Lipoxygenases are effective in the oxygenation of free PUFA (51). 15LO1 is highly expressed in human airway epithelial cells (52). A previous study revealed that the expression of $15 \mathrm{LO} 1$ is increased in Th2 inflammation observed in asthma (53). Moreover, airway epithelial cells isolated from patients with stable, non-exacerbating asthma have an elevated level of $15 \mathrm{LO} 1$, which is correlated with the fraction of exhaled nitric oxide, one of the key markers of Th2 inflammation (30). The present study detected the level of $15 \mathrm{LO} 1$ in the lung tissues of mice exposed to HDM, and an increase in the protein expression of 15LO1 was revealed, which corresponded with the results of previous literature.

As a component of the cystine/glutamate antiporter system $\mathrm{Xc}^{-}$, SLC7A11 is required for the exchange of extracellular cystine and intracellular glutamate across the plasma membrane, therefore providing adequate cystine within cells (54). Cystine is further reduced to cysteine within the cell, which is an important precursor required for GSH synthesis (55). Ferroptosis can be initiated by the depletion of cellular cysteine through inhibition of cystine uptake mediated by SLC7A11 $(56,57)$. Previous results have demonstrated that SLC7A11 downregulation significantly increases nascent neoplastic cell susceptibility to ferroptosis, thus keeping tumorigenesis in check $(42,43)$. In the present study, downregulation of SLC7A11 was detected in HDM-induced asthma, which is consistent with its role in ferroptosis. These data indicated that ferroptosis was observed in the HDM-induced mouse asthma model, and that ROS, GSH, GPX4, ACSL4, 15LO1 and SLC7A11 were involved in the ferroptosis of murine lungs following HDM-induced asthma (Fig. 4J).

There are certain limitations to the present study. Due to the limited sample volume and sample preparation, IL-4 levels in BALF and IgE levels in serum were not detected, which should be detected in future follow-up experiments. Furthermore, The effect of agonists and inhibitors of ferroptosis on airway inflammation in HDM-induced asthma also requires further study.

In summary, the present study demonstrated that AHR, airway inflammation, lipid peroxidation and ROS levels were increased in a murine model of HDM-induced asthma, and that HDM inhalation induced ferroptosis in the lungs. The results have helped to form an improved understanding of the pathogenesis of allergic asthma and targeted treatment strategies.

\section{Acknowledgements}

Not applicable.

\section{Funding}

The present study was supported by grants from The Young Elite Scientists Sponsorship Program by China Association for Science and Technology (grant no. 2018QNRC001), the National Natural Science Foundation of China (grant no. 82174495) and Shanghai Science and Technology Commission Project (grant no. 21S21902500).

\section{Availability of data and materials}

The datasets used and/or analyzed during the current study are available from the corresponding author on reasonable request.

\section{Authors' contributions}

YW and JD designed the study. WT and MD analyzed the data. WT, MD, FT, JC, XZ, WW, TW, JQ, LY and SW performed the experiments. YW and WT wrote the manuscript. All authors read and approved the final manuscript. YW and WT confirm the authenticity of all the raw data.

\section{Ethics approval and consent to participate}

The experimental procedures were approved by the Ethics Committee of Animal Experiments of Fudan University (authorization no. 2020-10-HSYY-WY-01; Shanghai, China). 


\section{Patient consent for publication}

Not applicable.

\section{Competing interests}

The authors declare that they have no competing interests.

\section{References}

1. Caraballo L, Zakzuk J, Lee BW, Acevedo N, Soh JY, Sánchez-Borges M, Hossny E, García E, Rosario N, Ansotegui I, et al: Particularities of allergy in the tropics. World Allergy Organ J 9: 20, 2016.

2. Abou-Hamdan M, Gharib B, Bajenoff M, Julia V and de Reggi M: Pantethine down-regulates leukocyte recruitment and inflammatory parameters in a mouse model of allergic airway inflammation. Med Sci Monit Basic Res 23: 368-372, 2017.

3. Rogliani P, Calzetta L, Matera MG, Laitano R, Ritondo BL, Hanania NA and Cazzola M: Severe asthma and biological therapy: When, which, and for whom? Pulm Ther 6: 47-66, 2020.

4. Calzetta L, Matera MG, Coppola A and Rogliani P: Prospects for severe asthma treatment. Curr Opin Pharmacol 56: 52-60, 2020.

5. Reddel HK, Bateman ED, Becker A, Boulet LP, Cruz AA, Drazen JM, Haahtela T, Hurd SS, Inoue H, de Jongste JC, et al: A summary of the new GINA strategy: A roadmap to asthma control. Eur Respir J 46: 622-639, 2015.

6. Sulaiman I, Greene G, MacHale E, Seheult J, Mokoka M, D'Arcy S, Taylor T, Murphy DM, Hunt E, Lane SJ, et al: A randomised clinical trial of feedback on inhaler adherence and technique in patients with severe uncontrolled asthma. Eur Respir J 51: 1701126, 2018.

7. Caraballo L: Mite allergens. Expert Rev Clin Immunol 13 297-299, 2017

8. Yang L and Zhu R: Immunotherapy of house dust mite allergy. Hum Vaccin Immunother 13: 2390-2396, 2017.

9. Custovic A, Green R, Smith A, Chapman MD and Woodcock A New mattresses: How fast do they become a significant source of exposure to house dust mite allergens? Clin Exp Allergy 26 . 1243-1245, 1996.

10. Fernández-Caldas E, Puerta L and Caraballo L: Mites and allergy. Chem Immunol Allergy 100: 234-242, 2014.

11. Li L, Qian J, Zhou Y and Cui Y: Domestic mite-induced allergy: Causes, diagnosis, and future prospects. Int J Immunopathol Pharmacol 32: 2058738418804095, 2018.

12. Jacquet A: Interactions of airway epithelium with protease allergens in the allergic response. Clin Exp Allergy 41: 305-311, 2011

13. Dolma S,Lessnick SL, Hahn WC and Stockwell BR: Identification of genotype-selective antitumor agents using synthetic lethal chemical screening in engineered human tumor cells. Cancer Cell 3: 285-296, 2003

14. Dixon SJ, Lemberg KM, Lamprecht MR, Skouta R, Zaitsev EM, Gleason CE, Patel DN, Bauer AJ, Cantley AM, Yang WS, et al: Ferroptosis: An iron-dependent form of nonapoptotic cell death Cell 149: 1060-1072, 2012.

15. Gao M, Yi J, Zhu J, Minikes AM, Monian P, Thompson CB and Jiang X: Role of mitochondria in ferroptosis. Mol Cell 73: 354-363.e3, 2019.

16. Tang D, Kang R, Berghe TV, Vandenabeele P and Kroemer G: The molecular machinery of regulated cell death. Cell Res 29: 347-364, 2019.

17. Friedmann Angeli JP, Schneider M, Proneth B, Tyurina YY, Tyurin VA, Hammond VJ, Herbach N, Aichler M, Walch A, Eggenhofer E, et al: Inactivation of the ferroptosis regulator Gpx4 triggers acute renal failure in mice. Nat Cell Biol 16: 1180-1191, 2014.

18. Wu X, Li Y, Zhang S and Zhou X: Ferroptosis as a novel therapeutic target for cardiovascular disease. Theranostics 11: 3052-3059, 2021

19. Ghiamati Yazdi F, Zakeri A, van Ark I, Leusink-Muis T, Braber S, Soleimanian-Zad S and Folkerts G: Crude turmeric extract improves the suppressive effects of lactobacillus rhamnosus GG on allergic inflammation in a murine model of house dust mite-induced asthma. Front Immunol 11: 1092, 2020.
20. Tibbitt CA, Stark JM, Martens L, Ma J, Mold JE, Deswarte K, Oliynyk G, Feng X, Lambrecht BN, De Bleser P, et al: Single-cell RNA sequencing of the Thelper cell response to house dust mites defines a distinct gene expression signature in airway Th2 cells. Immunity 51: 169-184.e5, 2019.

21. Wei Y, Dong M, Zhang H, Lv Y, Liu J, Wei K, Luo Q, Sun J, Liu F, Xu F and Dong J: Acupuncture attenuated inflammation and inhibited Th17 and treg activity in experimental asthma. Evid Based Complement Alternat Med 2015: 340126, 2015.

22. Wang S, Wuniqiemu T, Tang W, Teng F, Bian Q, Yi L, Qin J, Zhu X, Wei Y and Dong J: Luteolin inhibits autophagy in allergic asthma by activating PI3K/Akt/mTOR signaling and inhibiting Beclin-1-PI3KC3 complex. Int Immunopharmacol 94: 107460, 2021.

23. Myou S, Leff AR, Myo S, Boetticher E, Tong J, Meliton AY, Liu J, Munoz NM and Zhu X: Blockade of inflammation and airway hyperresponsiveness in immune-sensitized mice by dominant-negative phosphoinositide 3-kinase-TAT. J Exp Med 198: 1573-1582, 2003.

24. Gavino AC, Nahmod K, Bharadwaj U, Makedonas G and Tweardy DJ: STAT3 inhibition prevents lung inflammation, remodeling, and accumulation of Th2 and Th17 cells in a murine asthma model. Allergy 71: 1684-1692, 2016.

25. Honda K, Arima M, Cheng G, Taki S, Hirata H, Eda F, Fukushima F, Yamaguchi B, Hatano M, Tokuhisa T and Fukuda T: Prostaglandin D2 reinforces Th2 type inflammatory responses of airways to low-dose antigen through bronchial expression of macrophage-derived chemokine. J Exp Med 198: 533-543, 2003.

26. Yi S, Zhai J, Niu R, Zhu G, Wang M, Liu J, Huang H, Wang Y, Jing $\mathrm{X}$, Kang L, et al: Eosinophil recruitment is dynamically regulated by interplay among lung dendritic cell subsets after allergen challenge. Nat Commun 9: 3879, 2018.

27. Fransén-Pettersson N, Duarte N, Nilsson J, Lundholm M, Mayans S, Larefalk $\AA$, Hannibal TD, Hansen L, Schmidt-Christensen A, Ivars F, et al: A new mouse model that spontaneously develops chronic liver inflammation and fibrosis. PLoS One 11: e0159850, 2016.

28. Cheng D, Xue Z, Yi L, Shi H, Zhang K, Huo X, Bonser LR, Zhao J, Xu Y, Erle DJ and Zhen G: Epithelial interleukin-25 is a key mediator in Th2-high, corticosteroid-responsive asthma. Am J Respir Crit Care Med 190: 639-648, 2014.

29. Doll S, Proneth B, Tyurina YY, Panzilius E, Kobayashi S, Ingold I, Irmler M, Beckers J, Aichler M, Walch A, et al: ACSL4 dictates ferroptosis sensitivity by shaping cellular lipid composition. Nat Chem Biol 13: 91-98, 2017.

30. Telorack M, Meyer M, Ingold I, Conrad M, Bloch $\mathrm{W}$ and Werner S: A glutathione-Nrf2-thioredoxin cross-talk ensures keratinocyte survival and efficient wound repair. PLoS Genet 12: e1005800, 2016.

31. Ish P, Malhotra N and Gupta N: GINA 2020: What's new and why? J Asthma 58: 1273-1277, 2021.

32. Hogan AD and Bernstein JA: GINA updated 2019: Landmark changes recommended for asthma management. Ann Allergy Asthma Immunol 124: 311-313, 2020.

33. Wenzel SE: Asthma phenotypes: The evolution from clinical to molecular approaches. Nat Med 18: 716-725, 2012.

34. Agache I and Akdis CA: Precision medicine and phenotypes, endotypes, genotypes, regiotypes, and theratypes of allergic diseases. J Clin Invest 129: 1493-1503, 2019.

35. Ito R, Maruoka S, Soda K, Katano I, Kawai K, Yagoto M, Hanazawa A, Takahashi T, Ogura T, Goto M, et al: A humanized mouse model to study asthmatic airway inflammation via the human IL-33/IL-13 axis. JCI Insight 3: e121580, 2018.

36. Jäger A and Kuchroo VK: Effector and regulatory T-cell subsets in autoimmunity and tissue inflammation. Scand J Immunol 72: 173-184, 2010.

37. Shang XZ, Ma KY, Radewonuk J, Li J, Song XY, Griswold DE, Emmell $\mathrm{E}$ and $\mathrm{Li} \mathrm{L}$ : $\mathrm{IgE}$ isotype switch and $\mathrm{IgE}$ production are enhanced in IL-21-deficient but not IFN-gamma-deficient mice in a Th2-biased response. Cell Immunol 241: 66-74, 2006.

38. Gough L, Sewell HF and Shakib F: The proteolytic activity of the major dust mite allergen Der $p 1$ enhances the IgE antibody response to a bystander antigen. Clin Exp Allergy 31: 1594-1598, 2001.

39. Qiu S, Fan X, Yang Y, Dong P, Zhou W, Xu Y, Zhou Y, Guo F, Zheng Y and Yang JQ: Schistosoma japonicum infection downregulates house dust mite-induced allergic airway inflammation in mice. PLoS One 12: e0179565, 2017.

40. Lambrecht BN, Hammad H and Fahy JV: The cytokines of asthma. Immunity 50: 975-991, 2019. 
41. Bush A: Cytokines and chemokines as biomarkers of future asthma. Front Pediatr 7: 72, 2019.

42. Zou Y and Schreiber SL: Progress in understanding ferroptosis and challenges in its targeting for therapeutic benefit. Cell Chem Biol 27: 463-471, 2020.

43. Gao M and Jiang X: To eat or not to eat-the metabolic flavor of ferroptosis. Curr Opin Cell Biol 51: 58-64, 2018.

44. Krainz T, Gaschler MM, Lim C, Sacher JR, Stockwell BR and Wipf P: A mitochondrial-targeted nitroxide is a potent inhibitor of ferroptosis. ACS Cent Sci 2: 653-659, 2016.

45. Konstorum A, Tesfay L, Paul BT, Torti FM, Laubenbacher RC and Torti SV: Systems biology of ferroptosis: A modeling approach. J Theor Biol 493: 110222, 2020.

46. Lu SC: Regulation of glutathione synthesis. Mol Aspects Med 30: 42-59, 2009.

47. Dixon SJ and Stockwell BR: The role of iron and reactive oxygen species in cell death. Nat Chem Biol 10: 9-17, 2014.

48. Ingold I, Berndt C, Schmitt S, Doll S, Poschmann G, Buday K, Roveri A, Peng X, Porto Freitas F, Seibt T, et al: Selenium utilization by GPX4 is required to prevent hydroperoxide-induced ferroptosis. Cell 172: 409-422.e21, 2018.

49. Xu Y, Li X, Cheng Y, Yang M and Wang R: Inhibition of ACSL4 attenuates ferroptotic damage after pulmonary ischemia-reperfusion. FASEB J 34: 16262-16275, 2020.

50. Zhou Y, Liao J, Mei Z, Liu X and Ge J: Insight into crosstalk between ferroptosis and necroptosis: Novel therapeutics in ischemic stroke. Oxid Med Cell Longev 2021: 9991001, 2021.

51. Nicolaou A, Mauro C, Urquhart P and Marelli-Berg F: Polyunsaturated fatty acid-derived lipid mediators and T cell function. Front Immunol 5: 75, 2014.
52. Wenzel SE, Tyurina YY, Zhao J, St Croix CM, Dar HH, Mao G, Tyurin VA, Anthonymuthu TS, Kapralov AA, Amoscato AA, et al: PEBP1 wardens ferroptosis by enabling lipoxygenase generation of lipid death signals. Cell 171: 628-641.e26, 2017.

53. Zhao J, O'Donnell VB, Balzar S, St Croix CM, Trudeau JB and Wenzel SE: 15-Lipoxygenase 1 interacts with phosphatidylethanolamine-binding protein to regulate MAPK signaling in human airway epithelial cells. Proc Natl Acad Sci USA 108: 14246-14251, 2011.

54. Sun X, Ou Z, Chen R, Niu X, Chen D, Kang R and Tang D: Activation of the p62-Keap1-NRF2 pathway protects against ferroptosis in hepatocellular carcinoma cells. Hepatology 63: 173-184, 2016.

55. Schrier MS, Trivedi MS and Deth RC: Redox-related epigenetic mechanisms in glioblastoma: Nuclear factor (erythroid-derived 2)-like 2, cobalamin, and dopamine receptor subtype 4. Front Oncol 7: 46, 2017.

56. Xu X, Zhang X, Wei C, Zheng D, Lu X, Yang Y, Luo A, Zhang K, Duan X and Wang Y: Targeting SLC7A11 specifically suppresses the progression of colorectal cancer stem cells via inducing ferroptosis. Eur J Pharm Sci 152: 105450, 2020.

57. Zhang Y, Zhuang L and Gan B: BAP1 suppresses tumor development by inducing ferroptosis upon SLC7A11 repression. Mol Cell Oncol 6: 1536845, 2018 .

This work is licensed under a Creative Commons Attribution-NonCommercial-NoDerivatives 4.0 International (CC BY-NC-ND 4.0) License. 